\title{
ON WISHART AND NON-CENTRAL WISHART DISTRIBUTIONS ON SYMMETRIC CONES
}

\author{
EBERHARD MAYERHOFER
}

\begin{abstract}
Necessary conditions for the existence of non-central Wishart distributions are given. Our method relies on positivity properties of spherical polynomials on Euclidean Jordan Algebras and advances an approach by Peddada and Richards (1991), where only a special case (positive semidefinite matrices, rank one non-centrality parameter) is treated. Not only needs the shape parameters be in the Wallach set - as is the case for Riesz measures - but also the rank of the non-centrality parameter is constrained by the size of the shape parameter. This rank condition has been recently proved with different methods for the special case of symmetric, positive semidefinite matrices (Letac and Massam (2011) and Graczyk, Malecki and Mayerhofer (2016)).
\end{abstract}

\section{INTRODUCTION}

For specific parameter instances, the Wishart distribution arises as the distribution of the covariance of samples from the multivariate normal distribution and was discovered as such by Wishart [17. The Wishart distribution $\Gamma(\beta, \Sigma)$ on the cone of positive semidefinite $m \times m$ matrices $\mathcal{S}_{m}^{+}$, can be defined via its Laplace transform 1

$$
\Phi(u ; \beta, \Sigma):=\int_{\mathcal{S}_{d}^{+}} e^{-\operatorname{tr}(u \xi)} p(d \xi)=\operatorname{det}\left(I_{m}+2 u \Sigma\right)^{-\beta}, \quad u \in \mathcal{S}_{m}^{+},
$$

where $p \sim \Gamma(\beta, \Sigma)$. Here one assumes non-degenerate scale parameter $\Sigma \in \mathcal{S}_{m}^{++}$ (the positive definite matrices) and a non-negative shape parameter $\beta \geq 0$. From its definition, one can see that the Wishart distribution is a natural multivariate generalization of the Gamma distribution to the cone of positive semidefinite matrices. Unlike this one-dimensional distribution, the Wishart distribution does not exist for all natural parameter choices. In fact, it is well known that $\Gamma(\beta, \Sigma)$ exists, if and only if

$$
\beta \in \Lambda=\left\{0, \frac{1}{2}, \ldots, \frac{m-2}{2}\right\} \cup\left[\frac{m-1}{2}, \infty\right) .
$$

The set $\Lambda$ is often referred to as Gindikin set, in honor of Gindikin who studied Gamma distributions on homogeneous cones 8 .

Graczyk, Malecki and Mayerhofer [9] prove that in the more general case of the non-central Wishart distribution $\Gamma(\beta, \Sigma ; \Omega$ ) (with non-centrality parameter $\Omega \in$

2010 Mathematics Subject Classification. Primary 60B15; Secondary 05A10, 05A05,33C80.

Key words and phrases. Wishart Distribution, Jack Polynomials, Generalized Binomial Coefficients, Jack Polynomials, Symmetric Cones.

${ }^{1}$ The characteristic function as computed for half-integers $\beta$ by [14 p.444], and used in [15] as defining equation is cumbersome, due to the use of the complex power function $z^{\beta}, \beta>0$, and is therefore avoided throughout this paper. 
$\left.\mathcal{S}_{m}^{+}\right)$whose Laplace transform is given by

$$
\Phi(u ; \beta, \Sigma, \Omega)=\operatorname{det}\left(I_{m}+2 u \Sigma\right)^{-\beta} e^{-2 \operatorname{tr}\left(\Omega u \Sigma\left(I_{m}+2 u \Sigma\right)^{-1}\right)}, \quad u \in S_{m}^{+},
$$

in addition to (1.1), the rank condition must hold:

$$
\text { If } \beta<\frac{m-1}{2} \text {, then } \operatorname{rank}(\Omega) \leq 2 \beta \text {. }
$$

Their proof uses the modern theory of affine Markov semigroups with state space $\mathcal{S}_{m}^{+}$ [3], and studies the action of these on elementary symmetric functions. Another, perhaps more elementary but rather technical proof of Letac and Massam [11] requires the precise decomposition of $\Gamma\left(\frac{m-1}{2}, \Sigma ; \Omega\right)$ into its singular and absolute continuous parts, and a detailed analysis of the support of non-central Wishart distributions.

This paper advances the very first approach to the existence issue of non-central Wishart distributions by Peddada and Richards [15, Theorem 2] who use properties of spherical polynomials with matrix argument to derive the weaker condition (1.1), albeit only for the $\operatorname{rank}(\Omega)=1$ case. Besides, they were not aware of the rank condition (1.2), which had first been conjectured by the author in a talk at a CIMPA workshop in Hammamet in 2011 (this rank condition is termed "Mayerhofer conjecture" in [11] and "Non-central Gindikin set conjecture" in [9]).

The refinement of Peddada and Richards' method produces the shortest and most elementary proof of the statements in [9, 11]:

Theorem 1.1. Let $\beta \geq 0, \Omega \in \mathcal{S}_{m}^{+}$and $\Sigma \in \mathcal{S}_{m}^{++}$. If $\Gamma(\beta, \Sigma ; \Omega)$ exists, then conditions (1.1) and (1.2) must hold.

It turns out that Peddada and Richards' method is perfectly suited for an application in the general setting of symmetric cones. The appropriate generalization of Theorem 1.1 is developed in Section 4 (Theorem 4.10). This result offers also a solution to [15, Conjecture 1] in the Hermitian case (but goes beyond it, including a rank condition), while it of course comprises all other irreducible symmetric cones: The Lorentz cones and the positive self-adjoint cones of arbitrary dimensions (over the reals, complex numbers, quaternions) as well as the exceptional 27 dimensional cone of $3 \times 3$ matrices over the octonions.

Program. In Section 2 some facts on generalized binomial coefficients are elaborated, in particular their non-negativity, as well as strict positivity in certain cases (Theorem 2.3). These coefficients arise in expansions of zonal polynomials which in turn are normalized Jack polynomials, with a parameter depending on the geometry of the particular symmetric cone (Theorem 4.2). The above stated Theorem 1.1 is proved in Section 3.1 This result is then extended to the irreducible symmetric cone setting in Section 4 (Theorem 4.10). For the convenience of the reader, who might not be familiar with the algebraic structure of Euclidean Jordan Algebras, sections 3 and 4 are kept independent and self-contained.

In Appendix $\mathrm{A}$ some facts of symmetric cones, and algebraic properties for $\mathrm{Eu}-$ clidean Jordan algebras are summarized. Essential formulas for the so-called contiguous binomial coefficients are given in Appendix B Finally, in Appendix C] we elaborate the maximal domain of the moment generating function of Wishart distributions. Furthermore, the action of linear automorphisms as well as of the natural exponential family on non-central Wishart distributions, which is required for the proof of Theorem 4.10 is studied. 


\section{Positivity of Generalized Binomial Coefficients}

Let $m \in \mathbb{N}$, and $\sigma=\left(\sigma_{1}, \ldots, \sigma_{m}\right)$ be a multi-index. The degree of a multi-index $\sigma \in \mathbb{N}_{0}^{m}$ is $|\sigma|:=\sigma_{1}+\cdots+\sigma_{m}$. All multi-indices that we consider here are in non-increasing order. The length $l(\sigma)$ is the last index $i$ for which $\sigma_{i} \neq 0$. The set of length $m$ multi-indices is denoted by $\mathcal{E}_{m}:=\left\{\sigma \in \mathbb{N}_{0}^{m} \mid \sigma_{1} \geq \sigma_{2} \geq \cdots \geq \sigma_{m}\right\}$. The $i^{\text {th }}$ contiguous multi-index is defined by $\sigma^{i}:=\left(\sigma_{1}, \ldots, \sigma_{i}+1, \ldots, \sigma_{m}\right)$. Denote by $1^{m}$ the $m$-vector $(1, \ldots, 1)$.

For a given $\alpha>0$, the Jack polynomials $J_{\kappa}(; \alpha)$ indexed by $\kappa \in \cup_{m \geq 0} \mathcal{E}_{m}$ are the unique normalized symmetric polynomials in several variables with coefficients in the field $\mathbb{Q}(\alpha)$, which satisfy both a triangular, and an orthogonality property with respect to the a certain scalar product (for details, see [12, Chapter VI, (4.5)]). This paper uses another characterization put forward by R. Stanley (16, Theorem $3.1])$.

Theorem 2.1. The polynomial $J_{\kappa}(; \alpha)$ is an eigenfunction of the Laplace-Beltramitype operator

$$
D(\alpha):=\frac{\alpha}{2} \sum_{i=1}^{m} t_{i}^{2} \frac{\partial^{2}}{\partial t_{i}^{2}}+\sum_{\substack{1 \leq i, j \leq m \\ i \neq j}} \frac{t_{i}^{2}}{t_{i}-t_{j}} \frac{\partial}{\partial t_{i}},
$$

associated with the eigenvalue

$$
e_{\kappa}(\alpha):=\alpha \sum_{i=1}^{m} \kappa_{i} \frac{\left(\kappa_{i}-1\right)}{2}-\sum_{i=1}^{m}(i-1) \kappa_{i}+(m-1)|\kappa| .
$$

There are no further eigenfunctions linearly independent from the $J_{\kappa}$ 's.

Remark 2.2. 16] gives a formula in terms of conjugate indices. The quoted form in (2.1) can be obtained by using the computations in the proof of 14, Theorem 7.2.2] together with the proof of [16, Theorem 3.1], where one needs only to prove that $D_{\alpha} t_{1}^{\kappa_{1}} \ldots t_{m}^{\kappa_{m}}=e_{\kappa}(\alpha) t_{1}^{\kappa_{1}} \ldots t_{m}^{\kappa_{m}}+$ lower order terms.

The generalized binomial coefficients $\left(\begin{array}{c}\kappa \\ \sigma\end{array}\right)_{\alpha}$ are implicitly defined by the identity

$$
\frac{J_{\kappa}\left(t+1^{m} ; \alpha\right)}{J_{\kappa}\left(1^{m} ; \alpha\right)}=\sum_{s=0}^{k} \sum_{|\sigma|=s}\left(\begin{array}{c}
\kappa \\
\sigma
\end{array}\right) \frac{J_{\sigma}(t ; \alpha)}{J_{\sigma}\left(1^{m} ; \alpha\right)} .
$$

The coefficients (2.2) are rational numbers by construction. Peddada and Richards remark [15],

"In the real case no explicit formula is available for the generalized binomial coefficients, and it does not even appear to be known whether they are nonnegative always."

We will need more and less: It turns out that we need strict positivity, yet only for a special class of coefficients, the contiguous ones.

\footnotetext{
${ }^{2}$ The generalized binomial coefficients are well-defined, since the Jack polynomials $J_{\lambda}(; \alpha)$ with $\lambda_{1}+\lambda_{2}+\cdots=n$ form a basis of homogeneous symmetric functions of degree $n$ with coefficients in $\mathbb{Q}(\alpha)$ ([16], p.83). Note that in 2.2) the left side is a symmetric polynomial of the same degree as $J_{\kappa}(; \alpha)$ and the coefficients $J_{\kappa}\left(1^{m} ; \alpha\right)$ are strictly positive, see [16 Theorem 5.4].
} 
Theorem 2.3. $\left(\begin{array}{c}\kappa \\ \sigma\end{array}\right)_{\alpha}$ is a nonnegative rational number for any $\kappa, \sigma \in \mathbb{N}_{0}^{m}$. Furthermore, the contiguous coefficients $\left(\begin{array}{c}\sigma^{i} \\ \sigma\end{array}\right)_{\alpha}$ are strictly positive.

Proof. By [10, Lemma 4] (and for zonal polynomials, where $\alpha=2$, 14, Exercise 7.13]),

$$
\sum_{i=1}^{m}\left(\begin{array}{c}
\sigma^{i} \\
\sigma
\end{array}\right)_{\alpha}\left(\begin{array}{c}
\kappa \\
\sigma^{i}
\end{array}\right)_{\alpha}=(|\kappa|-|\sigma|)\left(\begin{array}{c}
\kappa \\
\sigma
\end{array}\right)_{\alpha} .
$$

If $|\kappa|=|\sigma|$, there is nothing to prove, due to the fact that (cf. [14, p. 268, (5)])

$$
\left(\begin{array}{l}
\kappa \\
\sigma
\end{array}\right)_{\alpha}=\delta_{\kappa \sigma} \in\{0,1\}
$$

The contiguous coefficients $\left(\begin{array}{c}\sigma^{i} \\ \sigma\end{array}\right)_{\alpha}$ can be explicitly computed for each $i=1, \ldots, m$. It follows immediately from the formula in Lemma B.2 below that

$$
\left(\begin{array}{c}
\sigma^{i} \\
\sigma
\end{array}\right)_{\alpha} \geq 0
$$

Induction over $d=|\kappa|-|\sigma|$ is used. For any $\kappa, \sigma$ and $d=0,1$, the statement holds, see (2.5), (2.4)). These cases form the induction basis.

Supposing the induction hypothesis

$$
\left(\begin{array}{l}
\mu \\
\nu
\end{array}\right)_{\alpha} \geq 0, \text { for all } \mu, \nu \text { satisfying }|\mu|-|\sigma| \leq d-1
$$

it follows that

$$
\left(\begin{array}{c}
\kappa \\
\sigma^{i}
\end{array}\right)_{\alpha} \geq 0
$$

for each $i=1, \ldots, n$, because $|\kappa|-\left|\sigma^{i}\right|=d-1$.

Hence by (2.3), (2.5) and (2.7),

$$
\left(\begin{array}{l}
\kappa \\
\sigma
\end{array}\right)_{\alpha}=\frac{1}{k-s}\left(\sum_{i=1}^{m}\left(\begin{array}{c}
\sigma^{i} \\
\sigma
\end{array}\right)_{\alpha}\left(\begin{array}{c}
\kappa \\
\sigma^{i}
\end{array}\right)_{\alpha}\right) \geq 0,
$$

and thus the first part of the statement is proved. The strict positivity of the contiguous coefficients follows directly from their explicit expressions provided by Lemma B.2.

\section{The Wishart Distribution on Positive Semidefinite Matrices}

Denote by $\mathcal{S}_{m}$ the symmetric $m \times m$ matrices.

Recall the definition of zonal polynomials of matrix argument. For a strictly positive constant $C_{\kappa}\left(I_{m}\right)$,

$$
C_{\kappa}(x)=C_{\kappa}\left(I_{m}\right) \Phi_{\kappa}(x), \quad \Phi_{\kappa}(x)=\int_{u \in \mathbb{O}(m)} \Delta_{\kappa}\left(u x u^{*}\right) d u, \quad x \in \mathcal{S}_{m},
$$

where $d u$ is the Haar measure on the orthogonal group $\mathbb{O}(m)$, and for $\kappa_{1} \in \mathcal{E}_{m}$

$$
\Delta_{\kappa}(x)=\Delta_{1}(x)^{\kappa_{1}-\kappa_{2}} \Delta_{2}(x)^{\kappa_{2}-\kappa_{3}} \ldots \Delta_{m}(x)^{\kappa_{m}},
$$

where $\Delta_{i}$ is the $i^{\text {th }}$ subminor of the matrix $x \in \mathcal{S}_{m}$.

Lemma 3.1. $C_{\kappa}(\Omega) \geq 0$. Furthermore, if $\operatorname{rank}(x)=k$ and $l(\kappa)=k$, then $C_{\kappa}(x)>$ 0 . 
Proof. $C_{\kappa}(\Omega) \geq 0$, because of the nonnegative integrand in the very definition of the zonal polynomials, eq. (3.1). Concerning strict positivity, note that the nonnegative map $u \mapsto \Delta_{\kappa}\left(u x u^{\top}\right)$ is strictly positive in a non-empty open neighborhood $U_{0}$ of the identity $u=I_{m}$. Since $\mathbb{O}(m)$ is a compact set, it can be covered by finitely many translates of $U_{0}$. The translation invariance of the Haar measure implies that strictly positive mass is assigned to $U_{0}$, whence

$$
\Phi_{\kappa}(x) \geq \int_{u \in \mathbb{O}(m) \cap U_{0}} \Delta_{\kappa}\left(u x u^{*}\right) d u>0 .
$$

Lemma 3.2. Let $\beta \geq 0, \Omega \in \mathcal{S}_{m}^{+}$and $\Sigma \in \mathcal{S}_{m}^{++}$. If the Wishart distribution $\Gamma(\beta, \Sigma ; \Omega)$ exists, so does $\Gamma(\beta, \Sigma ; t \Omega)$ exist for any $t \geq 0$.

Note that for $t=0$ the standard Wishart distribution is recovered.

Proof. [9, Lemma 3.5 (iii)] (replace $\Omega \leftrightarrow \Omega, \Sigma \leftrightarrow 2 \Sigma, \widetilde{\Omega}=t \Omega, \widetilde{\Sigma} \leftrightarrow 2 \Sigma$ ).

\subsection{Proof of Theorem 1.1.}

Proof. Suppose $\Gamma(\beta, \Sigma ; \Omega)$ exists. By Lemma 3.2 this is implies the existence of $\Gamma\left(\beta, I_{m} ; t \Omega\right)$, for each $t \geq 0$. Assume a random matrix $S(t) \sim \Gamma\left(\beta, I_{m} ; t \Omega\right)$, each $t \geq$ 0 , as random variables on a probability space $(\Omega, \mathcal{F}, \mathbb{P})$ with expectation operator $\mathbb{E}$.

Denote by $(a)_{\kappa}$ the "generalized Pochhammer symbol"

$$
(a)_{\kappa}:=\prod_{i=1}^{l(\kappa)}\left(a-\frac{1}{2}(i-1)\right)_{\kappa_{i}},
$$

where the classical Pochhammer symbol is used, which is defined for $b \in \mathbb{R}$ as

$$
(b)_{0}:=1, \quad(b)_{k}:=b(b-1) \ldots(b-k+1), \quad k \in \mathbb{N} .
$$

By [15, Equations (12)], for any $\kappa \in \mathcal{E}_{m}$ the following moment formula holds (note the slightly rewritten formula)

$$
\mathbb{E} C_{\kappa}(S(t))=C_{\kappa}\left(I_{m}\right) \sum_{\sigma}\left(\begin{array}{c}
\kappa \\
\sigma
\end{array}\right) \frac{(\beta)_{\kappa}}{(\beta)_{\sigma}} \frac{C_{\sigma}(t \Omega)}{C_{\sigma}\left(I_{m}\right)} .
$$

If $\beta \geq(m-1) / 2$, nothing need to be shown. Suppose therefore $\beta<(m-1) / 2$, and $k=\operatorname{rank}(\Omega)$. If $k=0$, then $\Omega=0$, and there is nothing to show. To outline a rigorous proof for [15, Theorem 2] of Peddada and Richards, and their result's extension including the rank condition, the rank one case is shown first: So assume $\operatorname{rank}(\Omega)=1$ and $m \geq 2$. For $\kappa=(1,1)=1^{2}, C_{\kappa}(\Omega)=0$, and therefore eq. (3.3) yields

$$
\begin{aligned}
\mathbb{E} C_{1^{2}}(S(t)) & =C_{1^{2}}\left(I_{m}\right)\left((\beta)_{1^{2}}+\frac{(\beta)_{1^{2}}}{(\beta)_{1}} \frac{C_{1}(t \Omega)}{C_{1}\left(I_{m}\right)}\right) \\
& =C_{1^{2}}\left(I_{m}\right)\left(\beta(\beta-1 / 2)+(\beta-1 / 2)\left(\begin{array}{c}
\kappa \\
1
\end{array}\right) \frac{C_{1}(t \Omega)}{C_{1}\left(I_{m}\right)}\right) .
\end{aligned}
$$

Since each zonal polynomail value is strictly positive (Lemma 3.1), $\beta \in[0,1 / 2$ ) is impossible, as it leads to strictly negative expectation, whence $2 \beta \geq 1=\operatorname{rank}(\Omega)$. 
For the general case $\operatorname{rank}(\Omega)=k$, consider $\kappa=1^{k+1}$. Then

$$
\begin{aligned}
\mathbb{E} C_{\kappa}(S(t))= & C_{\kappa}\left(I_{m}\right)\left((\beta)_{1^{k+1}}+(\beta)_{1^{k}} / \beta\left(\begin{array}{c}
\kappa \\
1
\end{array}\right) \frac{C_{1}(t \Omega)}{C_{1}\left(I_{m}\right)}+\ldots\right. \\
& \left.+(\beta-k / 2)\left(\begin{array}{c}
\kappa \\
1^{k}
\end{array}\right) \frac{C_{1^{k}}(t \Omega)}{C_{1^{k}}\left(I_{m}\right)}\right) .
\end{aligned}
$$

The last summand is the leading coefficient in $t$. Furthermore, the contiguous coefficient $\left(\begin{array}{c}\kappa \\ 1 k\end{array}\right)$ in this last summand is strictly positive, due to Lemma B.2. Hence $\beta \in[0, k / 2)$ implies that $\mathbb{E} C_{\kappa}(S(t))<0$, a mere impossibility. Whence $2 \beta \geq k$.

It remains to show that $2 \beta \in\{k / 2, \ldots,(m-2) / 2\}$. For small $t$, the zero order coefficient in (3.3) dominates all other coefficients, implying thus

$$
(\beta)_{\kappa} \geq 0
$$

for each $\kappa$. In particular, for each $1 \leq l \leq m$, and $\kappa=1^{l+1}$,

$$
(\beta)_{\kappa}=\beta(\beta-1 / 2) \ldots(\beta-l / 2) \geq 0,
$$

which is violated, unless $\beta \in\{k / 2, \ldots, m-2 / 2\}$ (for instance, if $\beta \in(k / 2,(k+$ $1) / 2$ ), take $l=k+1$, and thus $\left.(\beta)_{\kappa}<0\right)$.

\section{The Wishart Distribution on Symmetric Cones}

Let $C$ be an irreducible symmetric cone in a finite dimensional vector space $V$, $\operatorname{dim} V=n$. We denote by $r$ the rank of the associated simple Euclidean Jordan algebra, with unit element $e$, and let $d$ its Peirce invariant. The inner product on $V$ is chosen as $\langle x, y\rangle:=\operatorname{tr}(x y)$. For a brief review on symmetric cones see Appendix A.

The generalized Pochhammer symbol, parameterized in $\alpha>0$ (whose dependence is suppressed in the following) is given by

$$
(a)_{\kappa}:=\prod_{i=1}^{l(\kappa)}\left(a-\frac{1}{\alpha}(i-1)\right)_{\kappa_{i}},
$$

where the classical Pochhammer symbol is used (see eq. (3.2)).

4.1. Zonal Polynomials: Definition and basic properties. Let $K=O(V) \cap G$, where $\mathrm{O}(\mathrm{V})$ is the orthogonal group of $V$ and $G$ is the connected component of the identity in the linear automorphism group of $C$. Let $d k$ be the normalized Haar measure on the compact abelian group $K$. The spherical polynomials are defined by

$$
\Phi_{\kappa}(x):=\int_{K} \Delta_{\kappa}(k x) d k, \quad \kappa \in \mathcal{E}_{m}, \quad m \geq 1,
$$

where $\Delta_{k}$ denotes the generalized power (see Appendix A.3). Up to a postive constant 3

$$
c_{\kappa}=d_{\kappa} \frac{|\kappa| !}{\left(\frac{n}{r}\right)_{\kappa}}
$$

they are equal to the zonal polynomials:

$$
Z_{\kappa}(x)=c_{\kappa} \Phi_{\kappa}(x) .
$$

\footnotetext{
${ }^{3}$ The constant $d_{\kappa}=1 /\left\|\Delta_{\kappa}\right\|_{\Sigma}$ is computed explicitly in [7, Proposition XI.4.1 and Proposition XI.4.3].
} 
This normalization is tailored in such a way that

$$
\operatorname{tr}(x)^{k}=\sum_{|\kappa|=k} Z_{\kappa}(x)
$$

and thus [7, Proposition XII.1.3 (i)]

$$
e^{t r(x)}=\sum_{\kappa} d_{\kappa} \frac{d_{\kappa}}{\left(\frac{n}{r}\right)_{\kappa}} \Phi_{\kappa}(x), \quad x \in V .
$$

An immediate consequence of a functional relationship for zonal polynomials 7 , Corollary XI.3.2] is the following

\section{Lemma 4.1.}

$$
\int_{K} Z_{\kappa}\left(P\left(y^{1 / 2}\right) k x\right) d k=\frac{Z_{\kappa}(y) Z_{\kappa}(x)}{Z_{\kappa}(e)} .
$$

4.2. Zonal Polynomials are normalized Jack symmetric functions. The functions $Z_{\kappa}$ are special cases of normalized Jack polynomials, when considered as functions of the eigenvalues of $x$ :

Theorem 4.2. Let $\alpha=2 / d$, where $d$ is the Peirce invariant. Then for any $\kappa \in \mathcal{E}$,

$$
\Phi_{\kappa}(x)=\frac{J_{\kappa}(\lambda(x) ; 2 / d)}{J_{\kappa}\left(1_{l(\kappa)} ; 2 / d\right)}
$$

where $\lambda(x)=\left(\lambda_{1}, \ldots, \lambda_{r}\right)$ are the eigenvalues of $x$.

Example 4.3. For $d=1, k=2$ and $m=2, Z_{\kappa}=C_{\kappa}$, and

$$
\sum_{|\kappa|=k} C_{\kappa}(z)=\operatorname{tr}(z)^{k}=\left(\lambda_{1}+\lambda_{2}\right)^{2}=\left(\lambda_{1}^{2}+\lambda_{2}^{2}+\frac{2}{3} \lambda_{1} \lambda_{2}\right)+\frac{4}{3} \lambda_{1} \lambda_{2},
$$

where $\left(\lambda_{1}, \lambda_{2}\right)$ are the eigenvalues of the symmetric positive semidefinite matrix $z$. On the other hand, the Jack polynomials of degree 2 are given by ( $\underline{6}$, Table 1 , $\alpha=2]$ )

$$
J_{(1,1)}(\lambda ; \alpha=2)=2 \lambda_{1} \lambda_{2}, \quad J_{(2)}(\lambda ; \alpha=2)=3\left(\lambda_{1}^{2}+\lambda_{2}^{2}\right)+2 \lambda_{1} \lambda_{2} .
$$

By equation (4.6) the zonal polynomials are multiplies of the Jack polynomials, and by comparing (4.8) and (4.7) we must have the following relationship

$$
C_{(1,1)}=\frac{2}{3} J_{(1,1)}(, ; \alpha=2), \quad C_{(2)}=\frac{1}{3} J_{(2)}(, ; \alpha=2) .
$$

A consequence of Theorem 4.2 is the following crucial link between the generalized binomial coefficients in the context of symmetric cones, and those defined via Jack polynomials:

Remark 4.4. By the definition (4.1), we have $\Phi_{\kappa}(e)=1$. Therefore $c_{\kappa}=Z_{\kappa}(e)$. The generalized binomial coefficients of [7, p. 343], are defined by the relationship

$$
\Phi_{\mu}(e+x)=\sum_{|\nu| \leq|\mu|}\left(\begin{array}{l}
\mu \\
\nu
\end{array}\right) \Phi_{\nu}(x)
$$

Since $\Phi_{\kappa}(e)=1$, for each $\kappa$, we conclude that

$$
\left(\begin{array}{l}
\mu \\
\nu
\end{array}\right)=\left(\begin{array}{l}
\mu \\
\nu
\end{array}\right)_{2 / d}
$$


where the latter are the generalized binomial coefficients (2.2) for Jack polynomials of parameter $\alpha=2 / d$.

Proof of Theorem 4.2. By [7, Proposition VI.4.2], the Laplace-Beltrami operator is of the form

$$
\begin{aligned}
L \Phi_{\kappa}(x) & =\sum_{i=1}^{r} \lambda_{i}^{2} \frac{\partial^{2} \Phi_{\kappa}}{\partial \lambda_{i}^{2}}+d \sum_{i \neq j} \frac{\lambda_{i} \lambda_{j}}{\lambda_{i}-\lambda_{j}} \frac{\partial \Phi_{\kappa}}{\partial \lambda_{i}}+\frac{n}{r} \sum_{i=1}^{r} \lambda_{i} \frac{\partial \Phi_{\kappa}}{\partial \lambda_{i}} \\
& =\sum_{i=1}^{r} \lambda_{i}^{2} \frac{\partial^{2} \Phi_{\kappa}}{\partial \lambda_{i}^{2}}+d \sum_{i \neq j} \frac{\lambda_{i}^{2}}{\lambda_{i}-\lambda_{j}} \frac{\partial \Phi_{\kappa}}{\partial \lambda_{i}}+\left(\frac{n}{r}-(r-1) d\right) \sum_{i=1}^{r} \lambda_{i} \frac{\partial \Phi_{\kappa}}{\partial \lambda_{i}},
\end{aligned}
$$

where $\left(\lambda_{1}, \ldots, \lambda_{r}\right)$ are the eigenvalues of $x$. By the Euler equation for the homogeneous function $\Phi_{\kappa}$, we thus obtain

$$
L \Phi_{\kappa}-\left(\frac{n}{r}-(r-1) d\right)|\kappa| \Phi_{\kappa}=\frac{2}{\alpha} D(\alpha) \Phi_{\kappa}
$$

for $\alpha=2 / d$.

Furthermore, by [7, Exercise VII.2], $\Delta_{\kappa}$ is an eigenfunction of the LaplaceBeltrami operator, and therefore also $\Phi_{\kappa}$ is an eigenfunction of $L$, and by (4.9), it is an eigenfunction of $d D(2 / d)$ also. Since (the symmetrization of) the monomial $t_{1}^{\kappa_{1}} \ldots t_{m}^{\kappa_{m}}$ is the highest order term of $\Phi_{\kappa}$, and since (see the proof of [14, Theorem $7.2 .2]$ )

$$
d D(2 / d) t_{1}^{\kappa} \ldots t_{m}^{\kappa_{m}}=e_{\kappa}(\alpha) t_{1}^{\kappa_{1}} t_{1}^{\kappa} \ldots t_{m}^{\kappa_{m}}+\text { lower order terms, }
$$

its eigenvalue equals $e_{\kappa}(2 / d)$. Thus, by [16, Theorem 3.1],

$$
\Phi_{\kappa}=\sum_{\mu \leq \kappa} g_{\mu \kappa} J_{\mu}(; 2 / d) .
$$

By the second part of the proof of [16, Proposition 5.1], it follows that

$$
\Phi_{\kappa}(\cdot)=f_{\kappa}(d) J_{\kappa}(\cdot ; 2 / d),
$$

for some constant $f_{\kappa}(d)$.

By the definition (4.1), we have $\Phi_{\kappa}(e)=1$, hence

$$
f_{\kappa}(d)=J_{\kappa}\left(1_{l(\kappa)} ; 2 / d\right)^{-1} .
$$

Using Remark 4.4 we have the following generalization of [14, Theorem 7.6.3]:

\section{Proposition 4.5.}

$$
\Delta(A+e)^{-\beta} \int_{K} e^{-\left\langle\left(A^{-1}+e\right)^{-1}, k(\Omega)\right\rangle} d k=\sum_{k=0}^{\infty} \sum_{\kappa:|\kappa|=k}(-1)^{k} \frac{L_{\kappa}^{\beta}(-\Omega) Z_{\kappa}(A)}{k !},
$$

where the generalized Laguerre polynomials (indexed by $\kappa$ ) are defined by ( 7 , p.343])

$$
L_{\kappa}^{\beta}(x):=\sum_{\sigma}\left(\begin{array}{c}
\kappa \\
\sigma
\end{array}\right)_{2 / d} \frac{(\beta)_{\kappa}}{(\beta)_{\sigma}} \frac{Z_{\sigma}(-x)}{Z_{\sigma}(e)} .
$$

Proof. Use [7, Exercise XV.3] with $\Omega=-u, x=-A$ and the homogeneity of $Z_{\kappa}$ of degree $|\kappa|=k$. 
4.3. The Wishart distribution. The Wishart distribution on symmetric cones ([13, Corollary 3] and [1, (3.17)]) is defined as follows:

Definition 4.6. Let $\beta \geq 0$ and $\Sigma$ be a regular element in $C$. The Wishart distribution $\Gamma(\beta, \Sigma)$, if exists, is defined via its Laplace transform $\Delta(e+P(\sqrt{\Sigma} u))^{-\beta}$.

An immediate consequence of the positivity result on Riesz distributions on symmetric cones which have Laplace transforms of the form $\varphi_{\beta}(u)=\Delta(u)^{-\beta}$, is the following:

Proposition 4.7. The Wishart distribution $\Gamma(\beta, \Sigma)$ is a positive measure, if and only if $\beta$ belongs to the (so-called) Wallach set

$$
\Lambda:=\left\{0, \frac{d}{2}, \ldots, \frac{d}{2}(r-2)\right\} \cup\left[\frac{d}{2}(r-1), \infty\right) .
$$

We shall extend this result for the more general case of non-central Wishart distributions, including a rank condition on the shape parameter. In accordance with [4, Proposition 5.5], where this distribution arises naturally as time marginal of affine diffusion processes, we define the following

Definition 4.8. Let $\beta \geq 0$ and $\Sigma \in C^{\circ}$. The non-central Wishart distribution $\Gamma(\beta, \Sigma ; \Omega)$, if exists, is defined via its Laplace transform

$$
\int e^{-\operatorname{tr}(u \xi)} \Gamma(\beta, \Sigma ; \Omega)(d \xi):=\Delta(e+2 P(\sqrt{\Sigma}) u)^{-\beta} e^{-\left\langle\left(u^{-1}+2 \Sigma\right)^{-1}, \Omega\right\rangle} .
$$

Lemma 4.9. $Z_{\kappa}(\Omega) \geq 0$. Furthermore, if $\operatorname{rank}(x)=k$ and $l(\kappa)=k$, then $Z_{\kappa}(x)>$ 0 .

Proof. $Z_{\kappa}(\Omega) \geq 0$, because of the nonnegative integrand in the very definition of the zonal polynomials, eq. (4.1)- 4.3.

For the proof of strict positivity, note that the non-negative map $k \mapsto \Delta_{\kappa}(k x)$ is strictly positive in a non-empty open neighborhood $U_{0}$ of the identity map. Since $K$ is a compact set, it can be covered by finitely many translates of $U_{0}$. The translation invariance of the Haar measure implies that strictly positive mass is assigned to $U_{0}$, whence

$$
\Phi_{\kappa}(x) \geq \int_{u \in K \cap U_{0}} \Delta_{\kappa}(k x) d k>0 .
$$

Theorem 4.10. If $\Gamma(\beta, \Sigma ; \Omega)$ exists, then the following conditions must hold:

(i) $\beta \in \Lambda$, and

(ii) if $\beta \in\left\{0, \frac{d}{2}, \ldots \frac{d}{2}(r-2)\right\}$, then $d \operatorname{rank}(\Omega) \leq 2 \beta$.

Proof. If $2 \beta \geq d(r-1)$, nothing need to be shown. Assume therefore $2 \beta<d(r-1)$.

Suppose $S \sim \Gamma(\beta, \Sigma ; \Omega)$. Then by the second part of LemmaC.3 (i), $P\left(\sqrt{\Sigma^{-1}} S \sim\right.$ $\Gamma\left(\beta, e ; \Omega^{\prime}\right)$, where $\Omega^{\prime}=P\left(\sqrt{\Sigma^{-1}}\right)(\Omega)$ is of the exact same rank as $\Omega$, because $\Sigma$ is invertible. Second, by the first part of Lemma C.3 (i) using the map $P(\sqrt{s e})$, we obtain the existence of $\Gamma\left(\beta, t e ; s \Omega^{\prime}\right)$, for each $s>0$. By Lemma C.3 (ii) $\Gamma\left(\beta, e ; \frac{s \Omega^{\prime}}{s^{2}}\right)$ exists, for any $s>0$. Setting $s=t$, we see that $\Gamma\left(\beta, e ; t \Omega^{\prime}\right)$ exists for any $t>0$.

As the Laplace transforms converge pointwise for $t \rightarrow 0$ to $\operatorname{det}(e+2 u)^{-\beta}$, by Lévy's continuity theorem for Laplace transforms ([3, Lemma 4.5]) the limit is the Laplace transform of a probability measure (namely of $\Gamma(\beta, e)$ ). We conclude that 
$\Gamma\left(\beta, e ; t \Omega^{\prime}\right)$ exists for any $t \geq 0$. Let $(\Omega, \mathcal{F}, \mathbb{P})$ be a probability space that supports $S(t) \sim \Gamma\left(\beta, e ; t \Omega^{\prime}\right)$, for any $t \geq 0$, and denote by $\mathbb{E}$ the corresponding expectation operator.

Next, we establish a crucial moment formula: For any $\kappa \in \mathcal{E}$

$$
\mathbb{E} Z_{\kappa}(S(t))=Z_{\kappa}(e) L_{\kappa}^{\beta}(-t \Omega) .
$$

To this end, we manipulate the following sum, using Lemma 4.1 (second identity), equation (4.4) (third identity), Lemma A.1 (ii) (fourth identity),

$$
\begin{aligned}
& \sum_{k=0}^{\infty} \sum_{\kappa:|\kappa|=k}(-1)^{k} \frac{Z_{\kappa}(A) \mathbb{E}\left[Z_{\kappa}(S(t))\right]}{k ! Z_{\kappa}(e)}=\mathbb{E}\left[\sum_{k=0}^{\infty} \sum_{\kappa:|\kappa|=k}(-1)^{k} \frac{Z_{\kappa}(A) Z_{\kappa}(S(t))}{k ! Z_{\kappa}(e)}\right] \\
& =\mathbb{E}\left[\sum_{k=0}^{\infty} \sum_{\kappa:|\kappa|=k} \frac{(-1)^{k}}{k !} \int_{K} Z_{\kappa}\left(P\left(A^{1 / 2}\right) k S(t)\right) d k\right]=\mathbb{E}\left[\int_{K} e^{-\operatorname{tr}\left(P\left(A^{1 / 2}\right) k S(t)\right)} d k\right] \\
& =\mathbb{E}\left[\int_{K} e^{-\operatorname{tr}(A k S(t))} d k\right]=\int_{K} \mathbb{E}\left[e^{-\operatorname{tr}\left(k^{-1} A S(t)\right)} d k\right] \\
& =\int_{K} \Delta\left(e+k^{-1} A\right)^{-\beta} e^{-\left\langle\left(\left(k^{-1} A\right)^{-1}+e\right)^{-1}, t \Omega\right\rangle} d k \\
& =\Delta(e+A)^{-\beta} \int_{K} e^{-\left\langle\left(\left(k^{-1} A\right)^{-1}+e\right)^{-1}, t \Omega\right\rangle} d k \\
& =\Delta(e+A)^{-\beta} \int_{K} e^{-\left\langle\left(A^{-1}+e\right)^{-1}, k(t \Omega)\right\rangle} d k \\
& =\sum_{k=0}^{\infty} \sum_{\kappa:|\kappa|=k}(-1)^{k} \frac{L_{\kappa}^{\beta}(-t \Omega) Z_{\kappa}(A)}{k !},
\end{aligned}
$$

the last identity being Proposition 4.5. By comparison of coefficients, we conclude that (4.13) holds.

Let $r>2$ and $\operatorname{rank}(\Omega)=k \geq 0$, then for $\kappa=1^{k+1}$,

$$
\begin{array}{r}
\mathbb{E} Z_{\kappa}(S(t))=Z_{\kappa}(e)\left((\beta)_{1^{k+1}}+(\beta)_{1^{k}} / \beta\left(\begin{array}{c}
\kappa \\
1
\end{array}\right) \frac{Z_{1}(t \Omega)}{Z_{1}(e)}+\ldots\right. \\
\left.+(\beta-d k / 2)\left(\begin{array}{c}
\kappa \\
1^{k}
\end{array}\right) \frac{Z_{1^{k}}(t \Omega)}{Z_{1^{k}}(e)}\right) .
\end{array}
$$

The last summand is the leading coefficient in $t$. Furthermore, the contiguous coefficient $\left(\begin{array}{c}\kappa \\ 1^{k}\end{array}\right)$ in this last summand is strictly positive, due to Lemma B.2. Hence $\beta \in[0, d k / 2)$ implies that $\mathbb{E} Z_{\kappa}(S(t))<0$, a mere impossibility. Whence $d k \leq 2 \beta<$ $d(r-1)$.

It remains to show that $2 \beta \in\{d k, \ldots, d(r-2)\}$. For small $t$, the zero order coefficient in (4.13) dominates all other coefficients, implying thus

$$
(\beta)_{\kappa} \geq 0
$$

for each $\kappa$. In particular, for each $k+1 \leq l \leq r-1$, and $\kappa=1^{l+1}$,

$$
(\beta)_{\kappa}=\beta\left(\beta-d \frac{1}{2}\right) \ldots\left(\beta-d \frac{l}{2}\right) \geq 0,
$$

which is violated, if $\beta \in(d(l-1) / 2, d l / 2)$. 


\section{ApPendix A. BASIC PROPERTIES OF SYMMETRIC CONES}

Let $V$ be an $n$ dimensional vector space over $\mathbb{R}$, equipped with an inner product $\langle$,$\rangle and let C \subset V$ be a closed convex cone, with $C^{\circ}$ its interior. Let $G L(V)$ be the general linear group on $V$. $C$ is called homogeneous, if the linear automorphism group

$$
G\left(C^{\circ}\right):=\left\{g \in G L(V) \mid g\left(C^{\circ}\right)=C^{\circ}\right\}
$$

acts transitively on $C$, i.e., for each $c_{1}, c_{2} \in C$, there exists $g \in G\left(C^{\circ}\right)$ such that $g\left(c_{1}\right)=c_{2} . C$ is symmetric, if it is homogeneous and self-dual, i.e,

$$
C=\{x \in V \mid\langle x, u\rangle \geq 0 \text { for any } u \in C\} .
$$

Since irreducible symmetric cones can be associated with simple Euclidean Jordan algebras in a unique way ([7, Proposition III.4.5]), we identify the vector space $V$ with its Euclidean Jordan algebra, and $C$ is the set of squares, $C=\left\{x^{2} \mid x \in V\right\}$. Let therefore $C$ be a symmetric cone, and $e$ be an identity element in $V$.

For $x \in V$, the map $L(x): V \rightarrow V$ is the left multiplication $y \mapsto x y$. For $x \in V$, the quadratic representation $P(x): V \rightarrow V$ is then defined by

$$
P(x) y=2 L(x)(L(x) y)-L\left(x^{2}\right) y .
$$

In the case of associativity, we have due to commutativity of multiplication,

$$
P(x) y=x^{2} y=x y x .
$$

Therefore, the definition of $P$ in (A.1) reflects the fact that multiplication is not associative for all Euclidean Jordan algebras.

Clearly the set of squares is homogeneous, because for any $x \in C$, we can write $x=y^{2}$ for some $y \in V$, and $P(y) e=y^{2}=x$.

Let $\mathbb{R}[\lambda]$ be the ring of polynomials over $\mathbb{R}$, and for fixed $x \in V$ consider the ring $\mathbb{R}[x]$ of polynomials over $\mathbb{R}$, evaluated at $x$.

The minimal polynomial of $x$ is the polynomial with leading coefficient 1 that generates the ideal

$$
\mathcal{J}(x)=\{p \in \mathbb{R}[\lambda] \mid p(x)=0\},
$$

and then

$$
\mathbb{R}[x]=\mathbb{R}[\lambda] / \mathcal{J}(x) .
$$

The degree of the minimal polynomial of $x \neq 0$ is precisely

$$
m(x)=\min \left\{k>0 \mid\left\{e, x, \ldots, x^{k}\right\} \text { are linearly independent }\right\} .
$$

The rank of the Jordan algebra is defined as $r:=\max _{x \in V} m(x)$ and an element $x$ is called regular, if $m(x)=r$. The set of regular elements in $C$ is precisely the interior $C^{\circ}$ of the cone.

By [7, Proposition II.2.1], there exist unique polynomials $a_{1}, \ldots, a_{r}$, such that the minimal polynomial of every regular element is given by

$$
f(\lambda ; x)=\lambda^{r}-a_{1}(x) \lambda^{r-1}+\cdots+(-1)^{r} a_{r}(x),
$$

and we denote $\operatorname{det}(x)=a_{r}(x)$ and $\operatorname{tr}(x)=a_{1}(x)$, the determinant and trace of $x$. 
A.1. Determinant, Trace and Quadratic Representation. We use the trace as inner product by defining (any other symmetric bilinear form in a simple Euclidean Jordan algebra $V$ is a scalar multiple of the trace, see [7, Theorem III.4.1])

$$
\langle x, y\rangle:=\operatorname{tr}(x y) \text {. }
$$

This inner product is associative ([7, Proposition II.4.3]), that is,

$$
\langle x y, z\rangle=\langle y, x z\rangle \text {. }
$$

In other words: the left multiplication is a self-adjoint operator. The following are frequently used in this paper:

Lemma A.1. (i) $(P(x) y)^{-1}=P\left(x^{-1}\right) y^{-1}$,

(ii) $\langle P(x) y, z\rangle=\langle y, P(x) z\rangle$,

(iii) $\operatorname{det}(P(x) y)=\operatorname{det}\left(x^{2}\right) \operatorname{det}(y)$.

Proof. Property[(i) is [7, Proposition II.3.3 (ii)], and(iii) is [7, Proposition III.4.2(i)]. Property (ii) follows from the fact that $L(x)$ is a self-adjoint operator, (A.2).

A.2. Coordinates. The rank $r$ and the Peirce invariant $d$ of a symmetric cone are linked to two important coordinate systems on symmetric cones, which are introduced below for a better understanding of the paper (which is, however largely coordinate free).

A.2.1. Spectral Decomposition and rank. $f \in V$ is called idempotent, if $f^{2}=f$, hence $f \in C$. Two idempotents $f, g$ are orthogonal, if $f g=0$. An idempotent is primitive, if it is not the sum of two non-trivial idempotents. A set of idempotents is complete, if it sums to the unit element $e$. Any element $x \in V$ admits a spectral decomposition: There exists a complete set of mutually orthogonal primitive idempotents $\left\{e_{1}, \ldots, e_{r}\right\}$ (a so-called Jordan frame) and real numbers $\lambda_{1}, \ldots, \lambda_{r}$ such that $x=\lambda_{1} e_{1}+\cdots+\lambda_{r} e_{r}$. The coefficients $\lambda_{1}, \ldots, \lambda_{r}$ are called eigenvalues.

A.2.2. Pierce Decomposition II and invariant. Idempotents can only have eigenvalues $0,1 / 2$ or 1 . For $\lambda \in\{0,1 / 2,1\}$ and idempotents $c$, we define the linear subsaces $V(c, \lambda):=\{x \in V \mid c x=\lambda x\}$.

Let $c_{1}, \ldots, c_{r}$ be a Jordan frame. The space $V$ can be decomposed ([7, Theorem IV.2.1]) in the following orthogonal sum

$$
V=\bigoplus_{i \leq j} V_{i j}
$$

where $V_{i i}=V\left(c_{i}, 1\right)$ for $i=1, \ldots, r$, and for $1 \leq i<j \leq r, V_{i j}=V\left(p_{i}, 1 / 2\right) \cap$ $V\left(c_{j}, 1 / 2\right)$. Furthermore, the orthogonal projections $P_{i j}$ on $V_{i j}$ satisfy

$$
P_{i i}=P\left(c_{i}\right), \quad P_{i j}=4 L\left(c_{i}\right) L\left(c_{j}\right) .
$$

The dimension $d$ of $V_{i j}$, the Peirce invariant, is independent of $i, j$ and the Jordan frame. It satisfies ([7, Corollary IV.2.6])

$$
n=r+\frac{d}{2} r(r-1)
$$

For example, for $d=1$ (where $C$ is the space of positive semidefinite symmetric $r \times r$ matrices), we indeed have

$$
n=\frac{(r+1)}{2}=r+\frac{1}{2} r(r-1) .
$$


A.3. Generalized Power Functions and Orthogonal Group. For $\kappa \in \mathcal{E}_{m}$, and $m \leq r$, the generalized power functions are defined as

$$
\Delta_{\kappa}(x):=\Delta_{1}^{\kappa_{1}-\kappa_{2}}(x) \Delta_{2}^{\kappa_{2}}(x) \ldots \Delta_{m}(x)^{\kappa_{m}},
$$

where $\Delta_{j}$ for $j=1, \ldots, r$ denotes the principal minors corresponding to the Jordan algebras $V^{(j)}=V\left(c_{1}+\cdots+c_{j}, 1\right)=\left\{x \in V \mid x=x\left(c_{1}+\ldots c_{j}\right)\right\}$, where $\left(c_{1}, \ldots, c_{r}\right)$ is a Jordan frame. Clearly it holds that $\Delta_{\kappa}(x)=\lambda_{1}^{\kappa_{1}} \ldots \lambda_{m}^{\kappa_{m}}$, if $x=\lambda_{1} c_{1}+\ldots \lambda_{r} c_{r}$.

The spherical polynomials $\Phi_{\kappa}$ (and thus the zonal polynomials $Z_{\kappa}$ ) arise as symmetrizations of generalized powers, see (4.1). Let $O(V)$ be the orthogonal group defined by

$$
O(V):=\{g \in G L(V) \mid\langle g c, g c\rangle=\langle c, c\rangle \text { for all } c \in V\} .
$$

Let $G \subset G\left(C^{\circ}\right)$ be the connected component of the identity, and set $K:=$ $G \cap O(V)$. By [7, p.5] it already acts transitively on $C$. Furthermore, according to 7. Proposition I.1.9 and Proposition I.4.3], there exists an element $e \in C$ such that

$$
G\left(C^{\circ}\right) \cap O(V)=G\left(C^{\circ}\right)_{e}:=\left\{g \in G\left(C^{\circ}\right) \mid g e=e\right\},
$$

the latter denoting the stabilizer of $e$, and

$$
G_{e}=K:=G \cap O(V) .
$$

A nice result concerns the automorphism group $\operatorname{Aut}(V)$ (not to be confused with $G L(V))$, which is defined as

$$
\operatorname{Aut}(V):=\{A \in G L(V) \mid A(x y)=A(x) A(y)\} .
$$

By [7, Theorem III.5.1], we have

$$
\operatorname{Aut}(V)_{0}=K,
$$

where $\operatorname{Aut}(V)_{0}$ denotes the identity component of $\operatorname{Aut}(V)$.

A.4. Zonal polynomials and Symmetry. Since $K$ is a compact abelian group, there exists a Haar measure $d k$ on it. We use its unique normalization.

The zonal polynomials defined via (4.1) are symmetric functions, in the sense that they are $K$ invariant, by constructions. This implies the following:

Lemma A.2. $Z_{\kappa}(x)$ depend on the eigenvalues $\lambda_{1}, \ldots, \lambda_{r}$ of $x$ only.

Proof. Let $x, y \in V$, with Jordan frames $c_{1}, \ldots, c_{r}$ and $d_{1}, \ldots, d_{r}$ and eigenvalues $\lambda_{1}, \ldots, \lambda_{r}$ such that

$$
x=\sum_{i} \lambda_{i} c_{i}, \quad y=\sum_{j} \lambda_{j} d_{j} .
$$

By Theorem [7, Theorem IV.2.5] there exists an automorphism $A$ such that $A c_{i}=d_{i}$ for $i=1, \ldots, r$. Since $A$ is an algebra automorphism, and $\left(c_{i}\right)_{i=1}^{r}$ is a complete and orthogonal system of idempotents, we have

$A(e) A(e)=A\left(e^{2}\right)=A\left(\left(c_{1}+\ldots c_{r}\right)^{2}\right)=A\left(c_{1}^{2}+\cdots+c_{r}^{2}\right)=A\left(c_{1}+\cdots+c_{r}\right)=A(e)$

and therefore $A(e)=e$. Since Aut $\subseteq G L(V)$ by definition and $A(e)=e$, we conclude by (A.5) that $A \in K$. Hence by $K$-invariance, $Z_{\kappa}(x)=Z_{\kappa}(A x)=Z_{\kappa}(y)$. 


\section{Appendix B. Ferrers Diagrams}

The material of this section is a slight adaption of notation and results in [16, 10.

Any multi-index $\kappa=\left(\kappa_{1}, \ldots, \kappa_{m}\right)$ with $\kappa_{1} \geq \cdots \geq \kappa_{m}$ can be identified with its Ferrers diagram which is the specific partition

$$
\mathcal{P}_{\kappa}:=\left\{s=(i, j) \mid 1 \leq i \leq l(\kappa), 1 \leq j \leq \kappa_{i}\right\} .
$$

In this diagram, $\kappa_{i}$ are the number of squares in each row $i$. The number $\kappa^{j}$ of squares in each column $j$, where $1 \leq j \leq \kappa_{1}$, are

$$
\kappa^{j}=\#\left\{i \mid(i, j) \in \mathcal{P}_{\kappa}\right\} .
$$

For $s=(i, j) \in \mathcal{P}_{\kappa}$, write $i(s):=i$ and $j(s):=j$ to indicate its column or row label.

The arm-length, for each $s$ in the diagram, is defined as the number of squares to the right of $s$, that is

$$
a_{\kappa}(s):=\kappa_{i}-j(s)
$$

and the leg-length is the number of squares below $s$,

$$
l_{\kappa}(s):=\kappa^{j(s)}-i(s) .
$$

Definition B.1. The upper hook- length is defined by

$$
h_{\kappa}^{*}(s):=l_{\kappa}(s)+\alpha\left(1+a_{\kappa}(s)\right)
$$

and the lower hook-length is

$$
h_{*}^{\kappa}(s):=l_{\kappa}(s)+1+\alpha a_{\kappa}(s) .
$$

The following holds by [10, Proposition 2],

\section{Lemma B.2.}

where

$$
\left(\begin{array}{c}
\sigma^{i} \\
\sigma
\end{array}\right)_{\alpha}=j_{\sigma}(\alpha)^{-1} \prod_{s \in \mathcal{P}_{\sigma}} A_{\sigma^{i}} \prod_{s \in \mathcal{P}_{\sigma}} B_{\sigma^{i}}
$$

and

$$
j_{\sigma}(\alpha)=\prod_{s \in \mathcal{P}_{\sigma}} h_{\sigma}^{*}(s) \prod_{s \in \mathcal{P}_{\sigma}} h_{*}^{\sigma}(s)
$$

$$
A_{\sigma^{i}}=\left\{\begin{array}{l}
h_{\star}^{\sigma}(s), \text { if } j(s) \neq i, \\
h_{\sigma}^{*}(s) \quad \text { otherwise }
\end{array} \quad, \quad B_{\sigma^{i}}= \begin{cases}h_{\sigma^{i}}^{\star}(s), & \text { if } j(s) \neq i, \\
h_{*}^{\sigma^{i}}(s) & \text { otherwise. }\end{cases}\right.
$$

Note that in several formulas dependence on the $\alpha$ parameter is suppressed.

\section{Appendix C. Standardization of Wishart Distributions}

C.1. The moment generating function. This section shows that the Laplace transform (4.12) can be extended to its maximal domain, which is dictated by the blow up of the right side. Clearly, $\operatorname{det}(e+P(\sqrt{\Sigma})(u))>0$ for $u \in C$, and therefore the right side of (4.12) is well defined as long as $\operatorname{det}(e+P(\sqrt{\Sigma})(u))>0$. Since

$$
\begin{aligned}
\operatorname{det}(e+P(\sqrt{\Sigma})(u)) & =\operatorname{det}\left(P(\sqrt{\Sigma})\left(P\left(\sqrt{\Sigma^{-1}}\right) e+u\right)\right) \\
& =\operatorname{det}(\Sigma) \operatorname{det}\left(P\left(\sqrt{\Sigma^{-1}}\right) e+u\right)=\operatorname{det}(\Sigma) \operatorname{det}\left(\Sigma^{-1}+u\right),
\end{aligned}
$$

the right side of (4.12) is a real analytic function on the domain

$$
D_{\Sigma}:=-\Sigma^{-1} / 2+C^{\circ},
$$


but blows up as the argument $u$ approaches the boundary $\partial D_{\Sigma}$, since then the determinant vanishes for elements in $C$ that are not regular.

The following extends the validity of (4.12) to its maximal domain $D_{\Sigma}$ :

Proposition C.1. The Laplace transform of $\Gamma(\beta, \Sigma ; \Omega)$ can be extended to the domain $D_{\Sigma}$ and (4.12) holds for any $u \in D_{\Sigma}$.

For the proof of this statement we require the following concerning the analytical extension of the Laplace transform of a measure on the non-negative real line ( 9 , Lemma B.2]):

Lemma C.2. Let $\mu$ be a probability measure on $\mathbb{R}_{+}$, and $h$ an analytic function on $\left(-\infty, s_{1}\right)$, where $s_{1}>s_{0} \geq 0$ such that

$$
\int_{\mathbb{R}_{+}} e^{s x} \mu(d x)=h(s)
$$

for $s \in\left(-\infty, s_{0}\right)$. Then (C.1) also holds for $s \in\left(-\infty, s_{1}\right)$.

Proof of Proposition C.1. Let $\mu^{*}$ be the pushforward of $\mu=\Gamma(\beta, \Sigma ; \Omega)$ under $\xi \mapsto$ $\operatorname{tr}\left(\Sigma^{-1} \xi\right)=\operatorname{tr}\left(\left(P\left(\sqrt{\Sigma^{-1}} e\right) \xi\right)\right.$. Then $\mu^{*}$ is a probability measure on $\mathbb{R}_{+}$with Laplace transform

$$
\begin{aligned}
f(t): & =\int e^{t x} \mu^{*}(d x)=\int e^{-\operatorname{tr}\left(-t\left(P\left(\sqrt{\Sigma^{-1}}\right), \xi\right)\right.} \mu(d \xi) \\
& =(1-2 t)^{-\beta} e^{t(1-2 t)^{-1} \operatorname{tr}\left(\Sigma^{-1} \Omega\right)}
\end{aligned}
$$

and the right side is real analytic for $t<1 / 2$. Hence, by Lemma C.2 the left side is also finite for $t<1 / 2$ and equality holds in (C.2).

Therefore, we have shown that the formula (4.12) can be extended to $u=-t \Sigma^{-1}$, for any $t<1 / 2$. Since $u>-\Sigma^{-1} / 2$ implies $u>-t \Sigma^{-1}$ for some $t<1 / 2$, we have for any $u>-\Sigma^{-1} / 2$

$$
\int e^{-\operatorname{tr}(u \xi)} \mu(d \xi) \leq \int e^{t \operatorname{tr}\left(\Sigma^{-1} \xi\right)} \mu(d \xi)=f(t)<\infty
$$

and therefore the left side of (4.12) exists for any $u>-\Sigma^{-1} / 2$, as does the right side. The two real analytic functions (in several variables) agree on the connected open set $D_{\Sigma}$, since they do agree on the open subset $C^{\circ}([5,(9.4 .2)])$.

\section{C.2. Transformations and the Natural Exponential Family.}

Lemma C.3. Let $\beta \geq 0, \Omega \in C$ and $\Sigma \in C^{\circ}$.

(i) If $X \sim \Gamma(\beta, e ; \Omega)$, then $Y=P(\sqrt{\Sigma}) X \sim \Gamma(\beta, \Sigma ; P(\sqrt{\Sigma}) \Omega)$. Conversely, $Y \sim \Gamma(\beta, \Sigma ; P(\sqrt{\Sigma}) \Omega)$ implies $X=P\left(\sqrt{\Sigma}^{-1}\right) Y \sim \Gamma(\beta, e ; \Omega)$.

(ii) If $X \sim \mu(d \xi) \sim \Gamma(\beta$, te; $\Omega)$, then for $v:=\frac{1}{2}\left(-\frac{e}{t}+e\right)=\frac{1}{2}\left(1-\frac{1}{t}\right) e$ there exists a random variable $Y$ distributed as

$$
\nu(d \xi):=\frac{\exp (\operatorname{tr}(-v \xi)) \mu(d \xi)}{\mathbb{E}[\exp (\operatorname{tr}(-v X))]} \sim \Gamma\left(\beta, \frac{\Omega}{t^{2}} ; e\right) .
$$

Conversely, $\nu(d \xi) \sim \Gamma\left(\beta, e ; \frac{\Omega}{t^{2}}\right)$ implies that $\frac{e^{\operatorname{tr}(v \xi)} \nu(d \xi)}{\int e^{\operatorname{tr}(v \xi)} \nu(d \xi)} \sim \Gamma(\beta, t e ; \Omega)$. 
Proof. Proof of (i) Using repeatedly Lemma A.1 (i) and (iii), we get

$$
\begin{aligned}
\mathbb{E}\left[e^{-\operatorname{tr}(u Y)}\right] & =\mathbb{E}\left[e^{-\operatorname{tr}(u P(\sqrt{\Sigma}) X)}\right]=\mathbb{E}\left[e^{-\operatorname{tr}((P(\sqrt{\Sigma}) u) X)}\right] \\
& =(\operatorname{det}(e+2 P(\sqrt{\Sigma}) u))^{-\beta} e^{-\operatorname{tr}\left(\left(u^{-1}+P(\sqrt{\Sigma} e)^{-1} P(\sqrt{\Sigma}) \Omega\right)\right.},
\end{aligned}
$$

and since $P(\sqrt{\Sigma} e)=\Sigma$, we have indeed $Y \sim \Gamma(\beta, \Sigma ; P(\Sigma) \Omega)$. The second part can be proved quite similarly.

Proof of (ii) Note that due to Proposition C.1, $v \in D_{\Sigma}$, where $\Sigma=t e$ and (4.12) holds for $v$. Hence a random variable $Y$ with distribution $\frac{\exp (\operatorname{tr}(-v \xi)) \mu(d \xi)}{\mathbb{E}[\exp (\operatorname{tr}(-v X))]}$ exists. We compute its Laplace transform: First,

$$
e+2 P\left(\sqrt{\Sigma}(u+v)=e+2 P \sqrt{\Sigma} u-P(\sqrt{\Sigma}) \Sigma^{-1}+P \sqrt{\Sigma} e=P(\sqrt{\Sigma})(2 u+e),\right.
$$

because $P(\sqrt{\Sigma}) \Sigma^{-1}=2 \sqrt{\Sigma}\left(\sqrt{\Sigma} \Sigma^{-1}\right)-(\sqrt{\Sigma})^{2} \Sigma^{-1}=2 e-e=e$. Hence, by Lemma A.1 (ii) we have for $\Sigma=t e$,

$$
\operatorname{det}(e+2 P(\sqrt{\Sigma}(u+v))=t \operatorname{det}(2 u+e) .
$$

Second, we have

$$
\begin{aligned}
& \left\langle\left((u+v)^{-1}+2 t e\right)^{-1}, \Omega\right\rangle=\left\langle\left(\left(u+\frac{1}{2} e\left(1-\frac{1}{t}\right)\right)^{-1}+2 t e\right)^{-1}, \Omega\right\rangle \\
& \quad=\left\langle\left(u+\frac{1}{2} e\left(1-\frac{1}{t}\right)\left(e+2 t\left(u+\frac{1}{2} e\left(1-\frac{1}{t}\right)\right)\right)^{-1}, \Omega\right\rangle\right. \\
& =\left\langle\left(2 u+e-\frac{e}{t}\right)(e+2 u)^{-1}, \frac{\Omega}{2 t}\right\rangle \\
& =\left\langle\left((2 u+e)-\frac{1}{t}(2 u+e)+\frac{2 u}{t}\right)(e+2 u)^{-1}, \frac{\Omega}{2 t}\right\rangle \\
& =\frac{t-1}{2 t^{2}} \operatorname{tr}(\Omega)+\left\langle\left(u^{-1}+2 e\right)^{-1}, \Omega\right\rangle .
\end{aligned}
$$

The combination of the last computations for the trace, in combination with (C.3), yields

$$
\int e^{-\langle u+v, \xi} \mu(d \xi)=c(\Omega, t)\left(\operatorname{det}(u+2 e)^{-\beta} e^{-\left\langle\left(u^{-1}+2 e\right)^{-1}, \Omega\right\rangle}\right),
$$

with the constant $c(\Omega, t)=t^{-\beta} \exp \left(-\frac{t-1}{2 t^{2}} \operatorname{tr}(\Omega)\right)$. Since $\nu(d \xi):=\frac{\exp (\operatorname{tr}(-v \xi)) \mu(d \xi)}{\mathbb{E}[\exp (\operatorname{tr}(-v X))]}$ is a probability measure, by construction, and since the term within the brackets on the right side of (C.4) is the Laplace transform of $\Gamma\left(\beta, e ; \frac{\Omega}{t^{2}}\right)$, we conclude that $c(\Omega, t)=\mathbb{E}[\exp (\operatorname{tr}(-v X))]$, and thus $\nu(d \xi) \sim \Gamma\left(\beta, e ; \frac{\Omega}{t^{2}}\right)$, as claimed. The converse statement can be proved similarly.

\section{REFERENCES}

1. Muriel Casalis and Gérard Letac. The Lukacs-Olkin-Rubin characterization of Wishart distributions on symmetric cones. The Annals of Statistics, 24(2), 763-786, 1996.

2. Muriel Casalis and Gérard Letac. Characterization of the Jorgensen set in generalized linear models. Test, 3(1), 145-162, 1994.

3. Christa Cuchiero, Damir Filipović, Eberhard Mayerhofer and Josef Teichmann. Affine processes on positive semidefinite matrices. The Annals of Applied Probability, 21(2), 397-463, 2011.

4. Christa Cuchiero, Martin Keller-Ressel, Eberhard Mayerhofer, and Josef Teichmann. Affine Processes on Symmetric Cones. Journal of Theoretical Probability, 29(2), 359-422, 2016. 
ON WISHART AND NON-CENTRAL WISHART DISTRIBUTIONS ON SYMMETRIC CONESI7

5. Jean Dieudonné. Foundations of modern analysis. Pure and Applied Mathematics, P. Smith and S. Eilenberg, Eds. New York: Academic Press 10, 1969.

6. Ioana Dumitriu, Alan Edelman, and Gene Shuman. Mops: Multivariate orthogonal polynomials (symbolically). Journal of Symbolic Computation, 42(6), 587-620, 2007.

7. Jacques Faraut and Adam Korányi. Analysis on symmetric cones. Oxford: Clarendon Press, 1994.

8. Simon G Gindikin. Invariant generalized functions in homogeneous domains. Functional Analysis and its Applications, 9(1), 50-52, 1975.

9. Piotr Graczyk, Jacek Małecki, and Eberhard Mayerhofer. A Characterization of Wishart Processes and Wishart Distributions. Stochastic Processes and their Applications, 128(4), 13861404,2018

10. Jyoichi Kaneko. Selberg Integrals and Hypergeometric Functions Associated with Jack Polynomials. SIAM Journal on Mathematical Analysis, 24(4), 1086-1110, 1993.

11. Gérard Letac and Hélène Massam.The Laplace transform (det $s)^{-p} \exp \operatorname{tr}\left(s^{-1} w\right)$ and the existence of non-central Wishart distributions, Journal of Multivariate Analysis 163, 96-110, 2018.

12. Ian G. Macdonald. Symmetric Functions and Hall Polynomials. Oxford University Press, 1998.

13. Hélène Massam and Erhard Neher. On Transformations and Determinants of Wishart Variables on Symmetric Cones. Journal of Theoretical Probability, 10(4), 867-902, 1997.

14. Robb J. Muirhead. Aspects of Multivariate Statistical Theory, volume 197. John Wiley \& Sons, New York, 2005.

15. Shyamal Das Peddada and Donald St. P. Richards. Proof of a Conjecture of ML Eaton on the Characteristic Function of the Wishart distribution. The Annals of Probability, 19(2), 868-874, 1991.

16. Richard P. Stanley. Some Combinatorial Properties of Jack Symmetric Functions. Advances in Mathematics, 77, 76-115, 1989.

17. John Wishart. The Generalised Product Moment Distribution in Samples from a Normal Multivariate Population. Biometrika, 20A, 32-52, 1928.

Department of Mathematics and Statistics, University of Limerick, Castletroy, IreLAND

E-mail address: eberhard.mayerhofer@ul.ie 